\title{
Garantía del derecho humano de la salud al interior del centro regional de reinserción social Chilpancingo
}

\section{Guarantee of the human right to health inside the Chilpancingo regional social reinsertion center}

Garantia do direito humano à saúde dentro do centro regional de reintegração social de Chilpancingo

\section{Vera Judith Villa Guardiola}

veravilla@gmail.com

https://orcid.org/0000-0003-3222-3375

Universidad Autónoma de Guerrero, Guerrero, México

\section{Daniel Sánchez Romero}

08092573@uagro.mx

https://orcid.org/0000-0001-9555-1953

Universidad Autónoma de Guerrero, Guerrero, México

José Antonio Soto Sotelo

11461@uagro.mx

https://orcid.org/0000-0001-9898-5482

Universidad Autónoma de Guerrero, Guerrero, México

Artículo recibido en abril 2021 | Arbitraje en mayo 2021 | Aceptación en junio 2021 | Publicación en julio 2021

RESUMEN

\section{ABSTRACT}

\section{RESUMO}

La condición de aprisionamiento presupone un estado de vida que dista de comodidades, servicios, alejando la garantía del Estado en proteger los derechos humanos que les son consagrados a las personas sujetas a una sentencia en reclusión. El régimen penitenciario no ha logrado proteger la vida, dignidad e integridad de los sentenciados; por ejemplo, mediante un estudio socio jurídico se contrastaron las condiciones en que se presta el servicio de la salud, originando con ello una serie de violaciones a los derechos de dichas personas, concretamente, afecciones al derecho de la salud. Sucede así para el sector carcelario de Chilpancingo, Guerrero; ante tales inconsistencias, ¿Cómo puede protegerse el derecho humano de la salud? Las técnicas y métodos desarrollados harán constar la necesidad de velar por la protección de ese derecho y así evitar una adición a la pena impuesta al sentenciado.

Palabras clave: Chilpancingo; Derecho Humano; Enfermedades; Reclusión; Reinserción; Salud

The condition of imprisonment presupposes a state of life that is far from comforts and services, distancing the State's guarantee to protect the human rights that are consecrated to the persons subject to a prison sentence. The penitentiary system has not managed to protect the life, dignity and integrity of the sentenced persons; for example, through a socio-legal study, the conditions in which the health service is provided were contrasted, causing a series of violations to the rights of these persons, specifically, affections to the right to health. This is the case for the prison sector in Chilpancingo, Guerrero; in the face of such inconsistencies, how can the human right to health be protected? The techniques and methods developed will demonstrate the need to ensure the protection of this right and thus avoid adding to the sentence imposed on the convicted person.

Key words: Chilpancingo; Health, Human Right; Illnesses; Prison; Reinsertion

A condição de prisão pressupõe um estado de vida que está longe do conforto, dos serviços, distanciando a garantia do Estado na protecção dos direitos humanos que são consagrados às pessoas sujeitas a uma sentença em prisão preventiva. O sistema penitenciário não foi capaz de proteger a vida, dignidade e integridade das pessoas condenadas; por exemplo, através de um estudo sócio-legal, as condições em que o serviço de saúde é prestado foram contrastadas, caúsando uma série de violações aos direitos dessas pessoas, especificamente, afectos ao direito à saúde. É o caso do sector prisional em Chilpancingo, Guerrero; face a tais inconsistências, ¿como pode o direito humano à saúde ser protegido? As técnicas e métodos desenvolvidos mostrarão a necessidade de assegurar a protecção deste direito e assim evitar um acréscimo à pena imposta à pessoa condenada.

Palavras-chave: Chilpancingo; Direito Humano; Doenças; Prisão; Reinserção; Saúde 


\section{INTRODUCCIÓN}

La libertad de una persona es considerada como uno de los bienes jurídicos más importantes que una persona tiene, es un derecho que no debe condicionarse bajo ningún supuesto que atente contra ella o peligre con su afectación la integridad y dignidad de una persona, sin embargo en ocasiones, ésta no se vislumbra en su esplendor, ignorando los beneficios que se obtiene al hacer uso de ella como el libre esparcimiento, la elección de ir a determinado lugar, escoger el estilo de vida deseado; siendo esto bajo lo legalmente permitido y particularmente, adquirir servicios adecuados para satisfacer necesidades físicas y biológicas como el alimento, la salud u otros cuidados.

Es pues, que una persona en condición de libertad mantiene mayores posibilidades de gozar de aquellos derechos que le son consagrados por los altos órganos internacionales de protección a los derechos humanos y que son retomados por la constitución de cada nación, imponiendo la obligación a los distintos Estados prever las condiciones para que se materialicen dichos derechos. Sin embargo, no se distingue de tal manera al ubicarse dentro del panorama penitenciario o mejor conocido como "cárcel o prisión", dado que ese sector carcelario "se encuentra en una situación de mayor vulnerabilidad respecto del resto y que, por tanto, necesitan mecanismos especiales de protección" (Gutiérrez, 2013. p 6):

Con el panorama mostrado se trata de introducir al lector a fin de que en primer momento contraste aquel sector social que no se encuentra sometido a una situación de cautiverio y que gozan de diversos beneficios o facilidades de aquel otro que se halla bajo un internamiento en prisión por estar purgando una condena o sentencia; en palabras de Gutiérrez.

...todas las personas privadas de la libertad en centros de reclusión se encuentran en situación de vulnerabilidad. Su libertad está sujeta a la custodia y cuidado del Estado en un contexto frecuentemente permeado por desigualdades, abusos y opacidad. Aun en contextos en donde el abuso no es una constante, ha sido ampliamente demostrado que las condiciones en reclusión son lacerantes para la integridad física y mental. Esto se debe en gran medida a factores como el hacinamiento, la violencia, la falta de atención médica adecuada y el aislamiento de sus redes comunitarias y familiares (p. 6).

Sin embargo, no es intención del autor en ahondar en toda la gama de derechos que le son consagrados a las personas en calidad de sentenciados ni tampoco generalizar la problemática que posteriormente se expondrá, sobre las demás penitenciarias que existen a lo largo y ancho del país; y no porque no sea un punto débil sobre ellas o se les reste interés, sino que se invocará para este artículo lo referente al derecho humano de la salud, así como la necesidad de protegerlo en atención, cuidado y seguimiento en beneficio del centro regional de reinserción social de Chilpancingo Guerrero. 
Para continuar, se ha dejado en claro que la vida en prisión se caracteriza por no ser agradable, hay un gran abismo entre las comodidades y privilegios en comparación de quienes mantienen su plena libertad, por lo cual, es una situación en que las personas sentenciadas deberán de adaptarse prontamente dado que, será un régimen de vida en demasía gravoso ya que la hostilidad e inhumanismo, propio del régimen penitenciario, "propician condiciones carcelarias adversas para el respeto a la dignidad, la integridad y la y seguridad de las personas privadas de libertad" (Documenta, Asilegal, Instituto de Derechos Humanos Ignacio Ellacuría, SJ, 2012, p. 18).

Ahora bien, es importante decir que a rango constitucional, la salud es entendida como un derecho humano que requiere de toda la atención y protección por parte del Estado, tal como lo refiere el artículo primero de la Constitución Política de los Estados Unidos Mexicanos, siendo más puntual al tópico en comento, el artículo cuarto de dicha norma; a su vez, el arábigo dieciocho de la misma ley, cimienta al régimen penitenciario en el entendido de que las personas en el vinculadas, gozarán del respeto a sus derechos humanos intrínsecos, no obstante, no es así dado que se:

...evidencian situaciones de abstención u omisión en brindar una adecuada protección a la integridad física y psicológica de las personas privadas de la libertad, actos de tortura o tratos crueles, inhumanos o degradantes, así como la ausencia de condiciones adecuadas de estancia e instalaciones para vivir dignamente (2012, p. 18).

En esa tesitura, se considera que las cárceles se propicia un ambiente de violaciones a los derechos humanos que se perpetran a la luz de la complejidad del sistema penitenciario (2012) $y$, sin embargo, ninguna persona en reclusión o en libertad debe tolerar dada su condición de ser humano. Por ello, a pesar de que es responsabilidad del propio Estado velar por la garantía de los derechos humanos, éste no ha logrado satisfacer las necesidades propias de la persona sentenciada que lo mantenga dentro de los mínimos indispensables para su subsistencia, como lo es de un servicio de calidad para su salud.

Dicho de otra manera, es al Estado quien mediante su sistema penitenciario debe aportar a que se restituya el pleno ejercicio de las libertades el cual no significa volver a la persona al punto en que se encontraba antes de estar en prisión, sino que a través del régimen penitenciario se brinden las herramientas necesarias para que la persona sentenciada pueda regresar a la sociedad en mejores condiciones (Asilegal, 2018).

Ahora bien, es dable puntualizar qué se entiende por el derecho de la salud para luego relacionarlo y contrastarlo al marco de referencia que es el centro penitenciario de Chilpancingo; para ello, la Constitución contempla que en el aludido derecho: 
toda persona tiene derecho a la protección de la salud. La Ley definirá las bases y modalidades para el acceso a los servicios de salud y establecerá la concurrencia de la Federación y las entidades federativas en materia de salubridad general (Art. 4 Constitución Política de los Estados Unidos Mexicanos).

Del referido precepto constitucional se obtiene que no hay distinción alguna sobre quienes tienen derecho al derecho de la salud, sino que se pone en un mismo plano a los individuos a efecto de garantizar la protección de su salud. Por su parte, la Ley General de Salud, define tal concepto como un estado de completo bienestar físico, mental y social, y no solamente la ausencia de afecciones o enfermedades". (Artículo $1^{\circ}$ bis, 2020). Consecutivamente, el artículo segundo de la misma ley desglosa las finalidades del derecho a la salud como es el ofrecimiento de un bienestar físico y mental de la persona, mejoramiento de la calidad de vida, el disfrute de servicios de salud y de asistencia social que satisfagan eficaz y oportunamente las necesidades de la población, la promoción de la salud y la prevención de las enfermedades (Ley General de Salud, 2020).

De lo anterior, se infiere que todas las intervenciones que realiza el personal de salud así como las dependencias propias del Estado, están encaminadas a brindar atención al sector poblacional mediante cuidados e intervenciones a fin de preservar el derecho de la salud, lo cual repercutirá directamente sobre la vida, el cual cabe señalar, guarda relación directa al buen funcionamiento y desarrollo de una persona y sobre todo, por ser un bien jurídico tutelado; por ende, con la referida atención, deberá proteger, rehabilitar, curar y restaurar todo lo concerniente a la salud del sujeto que requiere de tales servicios. (Ley General de Salud, $\operatorname{art.32})^{1}$.

Seguidamente, es importante señalar la manera en que es contemplada la salud o el derecho humano de la salud a nivel constitucional e incluso, por los distintos órganos internacionales de derechos humanos; en ese entendido, por cuanto hace a la protección de los derechos humanos de las personas que yacen sometidas bajo un régimen carcelario, la Organización de las Naciones Unidas, por medio de la Comisión de Derechos Humanos, ha impuesto la obligación para los Estados parte, brindar un trato digno, es decir, atenciones, cuidados médicos, intervenciones necesarias para salvaguardar la salud de sus gobernados, incluyendo con esto a las personas privadas de la libertad ya que, al estar recluidos, se deduce que dichas personas, según Serrano Tárraga, se encuentran en una situación desfavorecida y de vulnerabilidad (2010, p. 557).

En esa virtud, la Ley Nacional de Ejecución Penal guarda relación directa con la protección del derecho de la salud ${ }^{2}$, aunadamente, el artículo 18 de la carta magna menciona que el sistema penitenciario se organizará sobre la base del respeto a los derechos humanos, y entre otras cosas confiere a la salud su importancia a efecto de concertar la reinserción del sentenciado, es

1 Artículo 32. Se entiende por atención médica el conjunto de servicios que se proporcionan al individuo, con el fin de proteger, promover y restaurar su salud. Para efectos del párrafo anterior los prestadores de servicios de salud podrán apoyarse en las Guías de Práctica Clínica y los medios electrónicos de acuerdo con las normas oficiales mexicanas que al efecto emita la Secretaría de Salud. $2 \quad$ Los artículos 9, 30, 31 y 32 de la LNEP, refieren los derechos de las personas privadas de la libertad en el entendido de que deben recibir asistencia médica, tratamientos que satisfagan sus necesidades relacionadas a su salud física, mental, moral, psicológica que garantice su integridad como ser humano. 
decir, devolverlo a la sociedad con miras a que mediante dicho derecho en conjunto con otros tópicos señalados por la norma en comento, la persona no reincida o vuelva a delinquir.

En ese tenor, se sostiene una vez más la obligación del Estado, quien mediante sus distintas instituciones o autoridades mantengan el ideal que, como garantes de derechos, establezcan medidas urgentes y pertinentes para que se materialice el derecho humano de la salud en las prisiones. Y es que, en la pertinencia de salvaguardar el referido derecho, se protege también la dignidad intrínseca de todo sentenciado, puesto que el internamiento infiere un deterioro en la integridad física, psíquica y moral (Gómez Reyes, 2019); por ejemplo, de la Rosa Rodríguez, opina que si el sentenciado al momento de obtener su libertad ha adquirido una enfermedad, esto se convierte en una adición a su pena, es decir, sufrió una depreciación de su condición física, en el que el Estado no garantizó este derecho fundamental (2016).

Dicha percepción muestra entonces un panorama en que el régimen penitenciario puede convertirse en un panorama que degrada el estado de salud de la persona que se encuentra compurgando una pena, entendiéndose esto como un daño a su estado mental, emocional, psicológico (Corte Interamericana de Derechos Humanos, 2020, p. 42).

Por otro lado, la Asamblea General de las Naciones Unidas aportó una serie de reglamentos y recomendaciones que apelan a favor de las personas que viven bajo los estándares de la prisión, por ejemplo, elaboró un reglamento para el tratamiento de los reclusos, llamadas "Reglas mínimas de las Naciones Unidas para el tratamiento de los reclusos”, mejor conocida como Reglas Nelson Mandela ${ }^{3}$ : mismo que preceptúa principios que deben desarrollarse al interior de las penitenciarías, apelando a favor de la dignidad humana, protección de la vida e integridad, no discriminación, mejores condiciones de vida, entre otras más.

Por cuanto al rubro de la salud, las mencionadas reglas enfatizaron una atención a la higiene, alimentación y servicios sanitario, con el objetivo de mantener en el mejor de las condiciones la dignidad humana propia de cada recluso y con ello contrarrestar situaciones discriminatorias o violaciones a derechos humanos (Jara Huérfano y Leonel Darío, 2017).

Con la mencionada norma se pretende, además de mejorar las políticas penitenciarias, reforzar las atenciones de asistencia médica y sanitaria al interior de los centros de reinserción social, para mejorar la salud integral de las personas recluidas ${ }^{4}$, satisfaciendo con ello la asistencia sanitaria relacionada a sus padecimientos o enfermedades.

En definitiva la ONU desarrolló en temas penitenciarios, referentes para el respeto de los derechos intrínsecos de las personas en situación de encarcelamiento, entre los cuales se ubica el acceso al servicio de salud; asimismo, la Comisión Interamericana de los Derechos Humanos, ha instaurado los principios y buenas prácticas sobre la protección de las personas privadas de la libertad en las Américas de 2008; mecanismo que refieren el respeto hacia la dignidad del reo,

3 Resolución 70/175. Reglas mínimas de las Naciones Unidas para el tratamiento de los reclusos (Reglas Nelson Mandela), 17 de diciembre de 2015

4 Reglas Nelson Mandela, regla 25. 
en atención a sus derechos humanos, a su vez, recalca la labor hacia los Estados la instauración de servicios acordes con el sistema de salud integral que sea continuo y preventivo.

Es por ello que todas las diversas disposiciones emitidas por los altos órganos internacionales, en concordancia con las legislaciones a nivel nacional y local en México, propugnan por la consagración del derecho de la salud como un derecho medular de las personas privadas de la libertad, que ofrezca un bienestar físico, mental y social a la par de la atención médica, psiquiátrica, ontológica con personal médico permanente y capacitado, con promoción de la salud, prevención y tratamiento de enfermedades infecciosas, endémicas y diversas con abastecimiento de medicamentos, en el que indudablemente el Estado debe garantizar toda clase de servicios idóneos y pertinentes para la atención de salud al interior de los centros penitenciarios.

Ahora bien, ha sido importante referir qué es lo que se ha dicho en el campo del régimen penitenciario y su relación con el derecho humano de la salud para adentrarse en la problemática que se plantea, dado que se podrá verificar que dichas pronunciaciones devienen de las problemáticas o carencias que se vive al interior de las penitenciarías y que advierten el incumplimiento del bagaje normativo y por ende, da a luz una serie de violaciones a los derechos de las personas sentenciadas, en este caso, el derecho de la salud.

En esa tesitura, se colige que las cárceles se encuentran en difíciles escenarios, o sea, sin ofrecer a las personas sentenciadas los mínimos indispensables para mantener un estilo de vida que no lacere su dignidad, reduciéndolo a humillaciones ante condiciones infrahumanas. En ese entendido, es que las personas sentenciadas del Centro regional de reinserción social de Chilpancingo, Guerrero, comparten la misma situación de vulnerabilidad e irrespeto por sus derechos, en este caso, la trasgresión a su derecho de la salud.

Para continuar, primeramente, se ha de hacer del conocimiento que en México, la Comisión Nacional de los Derechos Humanos, mantiene un programa de información penitenciaria en donde anualmente se elabora un diagnóstico nacional de supervisión penitenciaria mejor conocido por sus siglas DNSP, cuya finalidad es dotar de información respecto de la situación en que se encuentran las distintas penitenciarias en el país y reflejar la calidad de vida que lleva la población penitenciaria, dando con ello una serie de evaluaciones sobre los diferentes tópicos que conforma el sistema penitenciario.

Del aludido informe, para el año 2019, México caracterizó situaciones de vida en el ámbito carcelario muy pobre dado que reflejó situaciones que originan violaciones de derechos fundamentales, para el caso que nos ocupa, la salud ocupó uno de los rubros de mayor desgaste en su atención; es decir, que el diagnóstico permitió observar las carencias de falta de higiene, escasos programas de prevención de adicciones y desintoxicación y en general malos servicios de salud. (Observatorio de prisiones, Diagnóstico nacional de supervisión penitenciaria de la $\mathrm{CNDH})$. 
Sucede de igual manera en los centros penitenciarios a nivel federal, pues presentan fallas en los servicios de salud y atención sanitaria como problemas de abastecimiento de medicamentos, deficiencias en los programas de prevención, seguimiento y combate a las adicciones; incluyendo los relacionados con la falta de personal médico, deficiente atención, problemas de higiene en las diversas áreas, etcétera (CNDH, 2019).

Concatenado con el referido informe, la propia Comisión Nacional de los Derechos Humanos ha descrito la problemática que atraviesa el centro carcelario de Chilpancingo, situaciones que vulneran los derechos humanos, ya que a través de la recomendación 69/2019, de 23 de septiembre de 2019, exhortó al estado de Guerrero respecto de las condiciones en que se encontraban los centros carcelarios de dicha entidad, sin embargo, en lo que atañe al presente artículo, en el "cereso" de Chilpancingo, advirtió la situación de vida que lleva la población carcelaria de dicho lugar; panoramas tales como nula atención y protección al derecho de la salud, dado que éste se encuentra en un esquema de insalubridad, es decir, no hay condiciones para dar atenciones en espacios higiénicos.

Cabe señalar que es notable la necesidad de medicamentos al no contarse con ellos, de igual manera se distingue que los espacios de atención como consultorios médicos son escasos y no eficientes, así también, el personal médico encargado no está capacitado o especializado para atender a pacientes con enfermedades que requieren de mayor grado de diagnóstico, tampoco hay una distribución de alimentos saludables o dietas adecuadas para la población que así lo requiera (por ejemplo los enfermos diabéticos); es decir, se requiere disponibilidad de alimentos en cantidad y calidad suficientes para satisfacer las necesidades alimentarias de los individuos (Defensoría del pueblo, 2003, p. 94).

Es entonces que de las carencias que se han especificado, se tiene que dentro del centro de reinserción social referido habitan personas que requieren prioritaria atención por tratarse de adultos mayores con padecimientos crónicos degenerativos y distintas como de salud mental, cutánea, musculoesqueléticos, dental, metabólica, así como otro sector que requiere intervención por tener enfermedades venéreas, etc., (Pinzón Espita, y Meza Velandia, 2018).

Ante el ambiente de desatenciones a interior de los centros penitenciarios y de los reportes arrojados por la propia Comisión en el cual se puede ver la desprotección de los derechos humanos de las personas condenadas y desde luego, el informe ya especificado, arroja datos respecto de aquellas deficiencias del centro regional de reinserción social de Chilpancingo, por ende, se llega a la problemática de ¿Cómo garantizar el derecho de la salud de las personas sentenciadas del Centro de reinserción social de Chilpancingo Guerrero?, o sea, que ante las circunstancias ya mencionadas es notable que no se está brindando una protección del derecho humano de la salud.

Juntamente con lo anterior se interpreta que el apartado constitucional que narra la protección de las personas sentenciadas a través del régimen penitenciario no se está cumpliendo de manera puntual, con lo cual queda desfasada el ideal que el artículo primero de la misma 
constitución señala en el entendido de que el Estado se encuentra obligado de la protección, promoción, respeto y garantía de los derechos de las personas y entre los cuales están aquellos sujetos a una sentencia y en consecuencia en reclusión. A manera de detallada, se llega a la conclusión hasta este punto se ha desestimado el derecho humano a la salud ante actitudes de negligencia médica, falta de tratamiento de pacientes que necesitan de debida intervención o cuidados paliativos, asimismo escasa existencia en los suministros de medicamentos, servicios de traslados hospitalarios u hospitalización ante intervenciones urgentes, de igual manera no se les proporciona de artículos básicos para su higiene personal como son papel higiénico, pasta dental, jabón de baño (CNDH, recomendación No. 69/2019).

De manera concatenada, el mencionado centro de reinserción, según la recomendación elaborada por la CNDH, infiere que no se ha logrado garantizar el cuidado hacia la integridad física y moral del individuo ante la nula y debida separación de hombres y mujeres, lo cual trasgrede directamente en la esfera de sus derechos fundamentales ya que esto interfiere con una atención adecuada de acuerdo a su clasificación para con ello satisfacer sus necesidades según su género. (CNDH, recomendación No. 69/2019). Esto significaría que ante tales deficiencias sobre la prestación médica y en su conjunto en atención de los sentenciados, origina un menoscabo directo sobre la dignidad de dichas personas, es decir, se violentan sus derechos.

Por tales hechos, es de vital importancia que se intervenga de manera urgente dentro del centro de reinserción social de Chilpancingo, salvaguardar los objetivos del régimen penitenciario y con ello proteger la esfera de los derechos humanos de las personas que yacen en ese lugar al garantizar, respetar, promover y proteger su derecho a la salud5; lo cual debe ser realizado a través de la injerencia del mismo Estado puesto que es responsable del cuidado de la población carcelaria así como elaborar medidas emergentes para que se materialice el multicitado derecho.

\section{METODOLOGÍA}

Con motivo de la problemática detectada al interior del cereso de Chilpancingo, se ha fijado como objetivo proponer un programa que refuerce el derecho humano de la salud, es decir, que se alcance la protección establecida por los altos órganos de derechos humanos a fin de salvaguardar la integridad y dignidad de las personas sentenciadas en ese lugar, dado que, al detectar las fallas en dicho centro carcelario, se deduce un panorama en detrimento y de violencia a sus derechos; en consideraciones de la propia Corte IDH.

...las malas condiciones físicas y sanitarias de los lugares de detención pueden ser en sí mismas violatorias del artículo 5 de la Convención Americana, pues pueden causar sufrimientos de una intensidad que exceda el límite inevitable de sufrimiento que acarrea la detención, y porque conllevan sentimientos de humillación e inferioridad (2020, p.10). 
Seguidamente, se ha partido del estudio tipo socio-jurídico a fin de conocer el desarrollo o funcionamiento de la norma, su relación y efectividad contrastada en el centro de reinserción social de Chilpancingo Guerrero, sin embargo se ha logrado ver el impacto que las flaquezas del propio centro ha causado violaciones a derechos fundamentales, en este caso, sobre la salud; en la opinión de Giner, define en términos muy generales a la sociología del derecho como el campo de la investigación de los factores sociales que inciden en la organización, estructura, desarrollo y concepción del derecho y del Estado (1988, p. 733).

Por su parte, Rodríguez Lapuente la señala como una ciencia de carácter interdisciplinario, conformada por la sociología y por la denominada ciencia jurídica, partiendo del supuesto de que ambas son disciplinas muy diferentes entre sí: la ciencia jurídica es una ciencia prescriptiva y establece lo que se debe hacer y lo que no, mientras que la sociología observa directamente la realidad, registra los hechos y es empírica $(2001$, p.3).

Ante tales preceptos, se establece que los hechos sociales se relacionan con el derecho, en cómo son concebidos por la norma, en donde inclusive, convergen al mismo tiempo diversas áreas del conocimiento para establecer qué es lo socialmente permitido, en palabras del escritor, qué es lo que jurídicamente debe ser para con la sociedad. En relación con el presente tema, se espera que lo jurídico cause un impacto positivo en beneficio del derecho humano de la salud; que se pregone a favor de la población sentenciada en áreas de la salud, es decir, son los efectos que se espera cause la norma.

Por otro lado, el presente estudio se ha encaminado desde un nivel propositivo, el cual se fundamenta en una necesidad o vacío dentro de la institución, una vez que se tome la información descrita, se realizará una propuesta de sistema de evaluación del desempeño para superar la problemática actual y las deficiencias encontradas. Al identificar los problemas, investigarlos, profundizarlos y dar una solución dentro de un contexto específico (Metodología de la investigación, s.f.).

De lo anterior y en vista de la problemática que se ha originado alrededor del derecho humano de la salud, es bastante notable que existen vicios por parte de las autoridades penitenciarias al no instaurar un servicio idóneo en atenciones de salud o en salvaguarda de otros derechos, por lo tanto, es dable que para sanear tales deficiencias y al haberlos identificado puntualmente se originen soluciones certeras o mejor dicho, propuestas que corrijan los males causados por un mal seguimiento a los derechos humanos (la salud).

De manera equiparada, se ha visualizado la problemática desde el paradigma cuali-cuantitativo u holístico, el cual es definido:

...como la sinergia pertinente de técnicas, métodos, aproximaciones y conceptos tanto cuantitativos y cualitativos para dar respuesta al planteamiento de la investigación, abarca hacia un pluralismo metodológico que permite desarrollar una investigación a profundidad para una comprensión amplia de 
los objetos de estudio, a diferencia de trabajar de forma excluyente ambos enfoques (Carhuancho Mendoza., et al., (2019).

Dicho de otra manera, mediante la recopilación de datos se ha llegado a la interpretación de los mismos, es decir, datos referenciados sobre las condiciones en que se hallan las penitenciarías del país, particularmente la de Chilpancingo Guerrero, ya que por medio del diagnóstico nacional se da cuenta de las circunstancias que atraviesa el derecho humano de la salud, y en su comprensión e interpretación se detenta una urgente intervención para salvaguardar dicho derecho así como la dignidad humana de las personas recluidas bajo la condición de sentenciados en el referido centro.

Por cuanto hace al desarrollo del método inductivo- deductivo, entendido el primero de los citados como aquel que mediante el análisis de la información en hechos o acontecimientos particulares, permite llegar a conclusiones generales (Metodología de la investigación), por su parte el deductivo, a través de elementos generales como el análisis de las normas y demás tópicos, ayudará a definir una propuesta más adecuada (Metodología de la investigación, s.f.).

Es decir que, en la colaboración de dichos métodos, como se ha expuesto, se recolectó información que ayudara a tener un panorama de mayor comprensión; aparejado a la luz del tema en cuestión, el bagaje de la información que cita las distintas normas en materia de derechos humanos y de la salud, se elaboraron propuestas para mejorar las condiciones del citado derecho. Esto significa que fue indispensable la técnica documental para reunir una gama de información destacada para objetivizar el problema en cuestión y así aportar posibles soluciones.

Seguidamente, para la recolección de la información que fuere actual y pertinente para demostrar la hipótesis de la investigación lo fue la entrevista, misma que permitió partir de una guía prediseñada que contiene las preguntas que serán formuladas al entrevistado, para obtener la información requerida (Metodología de la investigación). Dicho de otra manera, se entabló una serie de diálogos que permitiera adquirir de las personas sentenciadas del multicitado centro penitenciario, testimonios, informes de primera mano al ser éste quien vive de cerca lo que acontece en ese ambiente carcelario.

\section{DISCUSIÓN}

De la recaudación de toda la información en comento como lo son los informes ofertados por la $\mathrm{CNDH}$, recomendaciones hechos por ese mismo órgano precursor de derechos humanos, diversa información atinente a la desprotección del derecho humano de la salud al interior de los distintos centros de reinserción social en México y particularmente el de Chilpancingo, Guerrero, se ha obtenido los resultados que a continuación se precisará: 
Es evidente que en la ciudad de Chilpancingo Guerrero, el sistema penitenciario se desarrolla bajo el denso ambiente de desprotección de los derechos humanos, originado por infraestructuras deficientes como consultorios médicos, sanitarios inadecuados, espacios para consumir alimentos y dormitorios deficientes, patios o áreas inadecuadas en donde puedan "recrearse o esparcirse en la medida de lo permitido, ha llegado al punto de que no se oferte a la población carcelaria lo indispensable para mejorar su condición humana, trayendo como consecuencia estrés, depresión, ansiedad, miedos y padecimientos mentales infringido por el hecho de estar recluido.

A su vez el hacinamiento de las personas sentenciadas en un solo lugar, promueva ambientes de enfermedades infecciosas o contagiosas,; por señalar algunas se tiene las de carácter o tipo sexual (sida, , problemas pulmonares (neumonía), tuberculosis, etc., agregado a ese problema, se cuenta con información de la mala alimentación para la población interna, deficiente cuidado y seguimiento a pacientes geriátricos, hipertensos, con alguna enfermedad terminal como cáncer, diabéticos, con esquizofrenia y muchos más.

Ahondando un poco más en salud alimentaria, se colige la falta de higiene con la que elaboran los alimentos e incluso del agua potable. Así también, indebida proporción en la alimentación para personas que necesiten un régimen alimentario adecuado a su estado de salud (Ley nacional de ejecución penal, articulo 9).

Otro problema detectado es no contar con programas pertinentes para anticiparse a problemas de salud en combate a adicciones, mucho menos programas constantes de promoción y seguimiento a la desintoxicación (CNDH, Recomendación 69/2019, p. 17). A la par, hay inconsistencias en cuanto a la falta de instalaciones especiales de atención de la salud, es decir, consultorios acondicionados para tal efecto, falta de insumos primarios que cubran aquellos siniestros dentro del centro, junto con ello, está la casi inexistencia de medicinas.

El personal médico no alcanza para atender a toda una población carcelaria y en ocasiones éste requiere estar mejor capacitado, incluso que cuente con alguna especialidad, a efecto de terminar con las comunes "atenciones médicas" desinteresadas en el que los pacientes son recetados genéricamente, pasados por una observación muy somera en donde en la mayoría de los casos, dan medicamentos para dispersar el dolor por tan solo un momento.

De todo lo dicho, se desprende que la calidad de vida de los de las personas sentenciadas al interior del Centro de reinserción social de Chilpancingo se posiciona en una situación infrahumana, de desprotección a la salud y omisiones a ese derecho, y que de no dar pronta solución, se estaría actualizando constantemente la violación a sus derechos fundamentales, sin que el régimen penitenciario pueda cumplir sus propósitos en cuanto a la salud; garantizar la integridad física y psicológica de las personas privadas de su libertad, como medio de proteger, promover y restaurar su salud (LNEP, Art. 74). 


\section{REFERENCIAS}

Asistencia legal por los derechos humanos. (2018). La situación del derecho a la reinserción social de las mujeres bajo la lupa de la ley nacional de ejecución penal. Recuperado de: asilegal. org.mx

Cámara de diputados del H. Consejo de la unión, Secretaría general, Ley General de Salud. (2020). Diario Oficial de la Federación.

Carhuancho Mendoza, I.M, et al. (2019). Metodología para la investigación holística. Guayaquil-Ecuador.

Comisión Nacional de los Derechos Humanos. (2019). Recuperado de https://www.cndh.org. $\mathrm{mx} / \mathrm{sites} /$ default/files/documentos/2019-04/ Com_2019_140.pdf

Corte Interamericana de Derechos Humanos. (2020). Cuadernillo No. 9: Personas privadas de libertad. p.42. Recuperado de https:// www.corteidh.or.cr/sitios/libros/todos/docs/ cuadernillo9.pdf

Constitución Política de los Estados Unidos Mexicanos. (2020)

CNDH México. (2018). Reglas mínimas de las naciones unidas para el tratamiento de los reclusos- Reglas Mandela.

Defensoría del pueblo. (2003). El derecho a la salud en la constitución, la jurisprudencia y los Instrumentos Internacionales. p. 94. Recuperado de https://www.corteidh.or.cr/ tablas/27803.pdf

De la Rosa Rodríguez, P.I. (2016). "El escenario y las reformas a la normatividad penitenciaria en el estado de San Luis Potosí. ¿Han sido suficientes ante la crisis de los penales en la entidad?" Ciencia jurídica, 5, (9), 7. Doi: https:// doi.org/10.15174/cj.v5i1.174

Diagnóstico Nacional de Supervisión Penitenciaria de la CNDH. Observatorio de Prisiones. Recuperado de https://observatorio-deprisiones.documenta.org.mx/archivos/14
Documenta, Asilegal, Instituto de Derechos Humanos Ignacio Ellacuría, SJ. (2012). Informe sobre la situación de las personas privadas de libertad en México.

Gómez Reyes, J.A. (2019). Los Derechos Humanos de las personas privadas de la libertad: Importancia y Aplicación. Revista mexicana de medicina forense. 4(2):57-74

Giner Salvador, y cols., Diccionario de sociología, Madrid, Alianza Editorial, 1988, p. 733.

Gutiérrez Román, J.L. (2013). Accesibilidad del derecho a la salud de los grupos de población en los centros de reclusión. Revista de derechos humanos- defensor. Derecho a la salud en centros de reclusión. 8, 6-21

Jara Huérfano, Leonel Darío. (2017). Estado Garante del derecho a la salud de las personas privadas de la libertad. Revista diálogos de saberes, (46), 2. Doi: https://doi. org/10.18041/0124-0021/dialogos.46.1595

Metodología de la investigación, s.f. p. 52. Recuperado de http://repositorio.utn.edu.ec/ bitstream/123456789/3548/4/CAP\%20III\%20 METODOLOGIA.pdf

Pinzón Espita, O.L y Meza Velandia, S. P. (2018). Prestación de servicios de salud de las personas privadas de la libertad. Imed pub journals. 14, (2:6). doi: 10.3823/1388

Rodríguez Lapuente, Miguel, Sociología del Derecho, 4ta. Ed. México, Porrúa, 2001, p. 3.

Serrano Tárraga, M.D. (2010). Derecho a la salud en los centros penitenciarios. Revista de derecho UNED. (7), p. 557 